\title{
Efficacy of Calcium Hydroxyapatite in Vocal Fold Augmentation for Unilateral Vocal Fold Paralysis in Asian
}

\author{
Doh Young Lee ${ }^{1}$, Eun-Jae Chung ${ }^{2}$ and Seong-Keun Kwon ${ }^{2}$ \\ ${ }^{I}$ Department of Otorhinolaryngology-Head and Neck Surgery, Seoul National University Boramae Medical Center, \\ Seoul National University College of Medicine, Seoul; and 2 Department of Otorhinolaryngology-Head and Neck Surgery, \\ Seoul National University Hospital, Seoul, Korea
}

\begin{abstract}
$=$ Abstract $=$
Background and Objectives: This study aimed to evaluate efficacy and complication of injection laryngoplasty using calcium hydroxyapatite (CaHA) for unilateral vocal fold paralysis in Asian. Materials and Methods : A prospective study was conducted on the adult patients with unilateral vocal fold paralysis from May 2015 through January 2016. Injection laryngoplasty was performed by one laryngologist. All patients underwent prospective voice evaluation using the subjective and objective comprehensive battery of assessments, before the procedure and after the procedure at 3 months, and 6 months. Results : A total of 7 patients ( 5 males and 2 females) were included in this study. VHI-10 was significantly decreased after injection laryngoplasty, at postoperative 6 months $(\mathrm{p}=0.031$ ), while VAS score and MDADI showed no difference. GRBAS scale gradually decreased in 3 months and 6 months follow-up without statistical significance. Acoustic analysis revealed that jitter, shimmer, and noise-to-harmonic ratio continuously decreased from the baseline at 3 months and 6 months, although statistical significance was not attained. In the aerodynamic analysis, maximal phonation time was gradually increased at 3 months and 6 months with significant difference ( $\mathrm{p}=0.016,0.031$, respectively). There was no side effect associated with the procedure. Conclusion : CaHA can be safely used in Asian patients and the onset of maximal efficacy seems to be slow than other studies with Caucasian patients.
\end{abstract}

KEY WORDS : Vocal fold paralysis · Calcium hydroxyapatite · Injection laryngoplasty.

\section{Introduction}

For successful injection laryngoplasty, followings are essential for injection materials: 1) being biologically inert, 2) immediate use with simple preparation, 3) no risk of infectious disease transmission, and 4) ability to be used with a fine-gauge needle. Because Calcium hydroxylapatite (CaHA) has proven to be a stable implant material with a minimal inflammatory response and no evidence of toxicity, it is one of the most frequently used material for injection laryngoplasty and has been formulated for vocal fold augmentation for glottal insufficiency. ${ }^{1,2)}$

An animal model of vocal fold injection using CaHA demonstrated an excellent immunologic response in the larynx

Received: May 10, 2018 / Revised: June 4, 2018

Accepted: October 16, 2018

Address for correspondence: Seong Keun Kwon, MD, PhD, Department of Otorhinolaryngology-Head and Neck Surgery, Seoul National University Hospital, 101 Daehak-ro Jongno-gu, Seoul 03080, Korea

Tel: (02) 2072-2286, Fax: (02) 745-2387, E-mail: otolarynx@hanmail.net for long-term follow-up as well as persistent vocal fold augmentation without negative impact on mucosal wave vibration. ${ }^{3)}$ In clinical studies, several reports demonstrated that rheologic properties of CaHA in commercial form is excellent in both short and long-term follow-up. ${ }^{4)}$ In addition, several complications have been also reported. ${ }^{5,6)}$ Nevertheless, there has been not enough evidence for Asian patients in terms of the efficacy, because most of studies were conducted in the western countries and most of participants are Caucasians. Facetem (CG Bio, Seongnam-si, Korea) is a newly developed CaHA for vocal fold augmentation. This study is a prospective study at a single medical center to evaluate the efficacy and safety of CaHA for the treatment of unilateral vocal fold paralysis of Asian patients.

\section{Materials and Methods}

\section{Study population}

From May 2015 through January 2016, 10 patients undergoing injection laryngoplasty using CaHA were prospectively 
included in this study. The inclusion criteria for enrolment were: 1) adult patients older than 20;2) glottal insufficiency due to unilateral vocal fold paralysis or vocal fold atrophy; and 3 ) other cause of glottal insufficiency with history of aspiration pneumonia or chronic aspiration. The enrolment exclusion criteria were: 1) history of laryngeal cancer; 2) history of previous injection laryngoplasty; 3) history of allergic reaction to $\mathrm{CaHA}$; 4) acute infection/inflammation or malignant lesion at injection site; 5) favourable prognosis in initial laryngeal EMG; and 6) refusal to be participated. The study protocol was approved by the Institutional Review Board (IRB) of the Seoul National University Hospital (IRB No. 1403-006-516).

\section{Study protocol and assessment of subjective/objective voice quality}

All patients were informed of the objective of the study and injection laryngoplasty procedure. At initial visit, following examination was performed: Storoboscopy, voice lab, larynx computed tomography, chest PA, laryngeal EMG (in case the duration of voice change is longer than 3 weeks). All patients underwent repeated functional evaluations before surgery and 3 months and 6 months postoperatively using a comprehensive battery of functional assessments. Voice handicap index $(\mathrm{VHI})^{7)}$ and MD Anderson Dysphagia Inventory (MDA$\mathrm{DI})^{8)}$ were surveyed at each visit. Voice evaluations were performed under consistent conditions by a single voice specialist. The voice specialist performed a perceptual rating of subjective voice assessments using the GRBAS scale. The GRBAS scale consists of the following five parameters: overall grade of hoarseness $(\mathrm{G})$, roughness $(\mathrm{R})$, breathiness (B), asthenia (A), and strain (S). A 4-point grading scale was used for each parameter, where $0=$ normal, $1=$ slight, $2=$ moderate, and $3=$ severe. Electroglottography was performed to evaluate the closed quotient (CQ). Acoustic voice evaluations were performed using the multi-dimensional voice program (MDVP) (Kay Elemetrics, Lincoln Park, NJ, USA). Aerodynamic analysis was performed using a spectrum analyser (Aerophone II Voice Function Analyzer; Kay Elemetrics Corp).

\section{Statistical analysis}

Results are presented as the mean value \pm standard devia- tion (SD) for continuous variables Continuous outcomes were analysed using Wilcoxon signed rank test. All statistical analyses were performed using IBM SPSS Statistics for Windows, Version 20.0. (IBM Corp., Armonk, NY, USA) Pvalues $<0.05$ were considered statistically significant.

\section{Results}

\section{General characteristics}

Among 10 patients who were initially enrolled in this study, a total of 7 patients ( 5 males and 2 females) were included and 3 patients were excluded due to insufficient initial screening data. Mean age was $69.0 \pm 8.9$ and all of the patients had unilateral vocal fold paralysis. Trans-cricothyroid membrane approach was performed on 6 patients $(85.7 \%)$ and trans-thyrohyoid membrane approach on 1 patients (14.3\%). Six patients were left unilateral vocal fold paralysis. Etiology of vocal fold paralysis was nerve injury during the thoracic surgery in 5 patients, idiopathic in 1 patient, and lung cancer in 1 patient. During the injection laryngoplasty procedure, exposure of the larynx was generally good in 6 patients, all injection were done in deep musculature. All procedure were performed by one laryngologist (SKK) and the surgeon's rating on the difficulty of injection was mostly low.

\section{Analysis of voice quality}

VHI-10 was significantly decreased after injection laryngoplasty, at postoperative 6 months $(\mathrm{p}=0.031)$, while VAS score and MDADI showed no difference (Table 1). Stroboscopy showed that all of the parameters, mucosal wave, glottal closure, symmetry, and periodicity, were improved after injection laryngoplasty while statistical significance was not attained.

GRBAS scale gradually decreased in 3 months and 6 months follow-up without statistical significance. CQ was not significantly different at postoperative 3 and 6 months. Acoustic analysis revealed that jitter, shimmer, and noise-to-harmonic ratio continuously decreased from the baseline at 3 months and 6 months, although statistical significance was not attained ( $\mathrm{p}=0.063,0.125$, respectively) (Table 2 ). In aerodynamic analysis, maximal phonation time was gradually increased at 3 months and 6 months with significant difference (base-

Table 1. Subjective outcomes of voice and swallowing

\begin{tabular}{llcccc}
\hline & Baseline & 3 months & p-value* & 6 months & p-value $^{\dagger}$ \\
\hline VAS & $31.71 \pm 13.92$ & $58.57 \pm 24.78$ & 0.078 & $68.33 \pm 17.22$ & 0.125 \\
VHI-10 & $24.43 \pm 7.70$ & $13.71 \pm 10.75$ & 0.063 & $6.50 \pm 4.55$ & 0.031 \\
MDADI & $68.57 \pm 30.24$ & $62.86 \pm 29.28$ & 0.750 & $86.67 \pm 10.33$ & 0.500 \\
\hline
\end{tabular}

* : comparison between baseline and 3 months, $\dagger$ : comparison between 3 and 6 months. VHI : voice handicap index, MDADI : MD Anderson dysphagia inventory 
Table 2. Change in GRABS : Closed quotients, and acoustic analysis

\begin{tabular}{lccccc}
\hline & Baseline & 3 months & p-value* & 6 months & $p_{\text {-value }}^{\dagger}$ \\
\hline GRABS & $2.43 \pm 0.53$ & $1.43 \pm 0.98$ & 0.125 & $0.83 \pm 0.98$ & 0.063 \\
EGG-CQ & $56.49 \pm 0.22$ & $43.00 \pm 5.40$ & 0.500 & $43.62 \pm 6.94$ & 0.500 \\
Acoustic analysis & & & & \\
FO & $155.63 \pm 49.82$ & $150.00 \pm 42.45$ & 0.688 & $145.90 \pm 47.66$ & 0.813 \\
Jitter & $7.28 \pm 2.87$ & $3.63 \pm 2.95$ & 0.156 & $1.31 \pm 1.14$ & 0.063 \\
Shimmer & $14.05 \pm 5.72$ & $7.36 \pm 4.72$ & 0.156 & $5.48 \pm 2.68$ & 0.125 \\
PPQ & $5.48 \pm 4.15$ & $2.26 \pm 1.86$ & 0.156 & $0.75 \pm 0.62$ & 0.063 \\
APQ & $11.16 \pm 4.75$ & $5.44 \pm 3.53$ & 0.125 & $4.28 \pm 2.01$ & 0.250 \\
NHR & $0.40 \pm 0.25$ & $0.17 \pm 0.06$ & 0.063 & $0.14 \pm 0.02$ & 0.063 \\
\hline
\end{tabular}

EGG-CQ : electroglottography-closued quotients, $P P Q$ : pitch perturbation quotient, APQ : amplitude perturbation quotient, NHR : noise to harmonic ratio

Table 3. Change in aerodynamic study

\begin{tabular}{lccccc}
\hline & Baseline & 3 months & p-value* & 6 months & p-value $^{\dagger}$ \\
\hline SPL & $69.49 \pm 6.36$ & $77.73 \pm 8.51$ & 0.078 & $82.48 \pm 2.67$ & 0.031 \\
SGPmean & $11.97 \pm 3.56$ & $12.50 \pm 5.43$ & 0.813 & $10.65 \pm 2.39$ & 0.438 \\
SGPpeak & $13.54 \pm 4.03$ & $13.74 \pm 5.72$ & 1.000 & $11.61 \pm 2.68$ & 0.313 \\
MPT & $2.45 \pm 1.18$ & $9.64 \pm 7.01$ & 0.016 & $13.24 \pm 7.15$ & 0.031 \\
Voice efficiency & $65.55 \pm 108.53$ & $742.26 \pm 1520.82$ & 0.156 & $886.70 \pm 1110.64$ & 0.063 \\
MFR & $367.14 \pm 218.84$ & $210.00 \pm 176.73$ & 0.016 & $295.00 \pm 324.88$ & 0.313 \\
\hline
\end{tabular}

SPL : sound pressure level, SGP : subglottic pressure, MPT : maximal phonation time, MFR : mean flow rate

line $2.45 \pm 1.18,3$ months $9.64 \pm 7.01,6$ months $13.24 \pm 7.15)(\mathrm{p}=$ $0.016,0.031$, respectively) (Table 3).

\section{Side effects}

One patients showed hyperemia of larynx, while it was more related with the injection laryngoplasty procedure itself or respiratory tract infection than side effect or allergic reaction to the injection material. Larynx hyperemia was resolved at 3 month postoperatively. There was no other side effect related to the injection material.

\section{Discussion}

This study demonstrated that newly developed CaHA showed comparable efficacy to the other formula of CaHA in injection laryngoplasty. Enhancing quality of life by creating a more effective and understandable voice is the primary purpose of phonosurgical vocal fold injection. Thus, a patient's subjective experience of improvement is the most important measure of a procedure's efficacy. In this short-term trial, injection with the CaHA produced successful results, with all fully evaluated patients reporting an improvement in voice quality. Supporting these self-evaluations were the aerodynamic results comparing mean airflow rates before and after injection.

Previous studies showed that subjective and objective voice outcomes continuously improved until postoperative 3 months and those became worse or similar after 3 months. ${ }^{9)}$ On contrast to the previous reports with Caucasian patients, our study showed that CaHA has longer effect as the subjective and most of objective outcomes improved till 6 months after the injection laryngoplasty. VHI score of unilateral vocal fold paralysis in previous reports has been shown to be as high as 25 , and decreased as low as around 15 after injection laryngoplasty. Although Carroll et al. reported a long-term clinical follow-up result after CAHA injection laryngoplasty, according to the patients' subjective report, and concluded that the average length of benefit was 18.6 months, the maximal benefit was observed at around 3 months in most of the western papers. ${ }^{10)}$ In contrast, our study showed that VHI score at postoperative 6 months was even lower than 3 months although none of the participants in our study showed recovery of unilateral vocal cord paralysis during the 6 months follow-up. In addition, jitter, shimmer, pitch perturbation quotient, noise to harmonic ratio, and maximal phonation time gradually improved until 6 months.

For long-term or permanent injection laryngoplasty, CaHA might be the best candidate. Finding an ideal long-term injectable for vocal fold augmentation has been the objectives of those who treat vocal fold paralysis. After using the paraffin as injectable material, many currently available materials such as autologous fat, fascia, hyaluronic acid, and collagen-based 
products showed limitation of biodegradable and loss of efficacy in long-term. ${ }^{11-13)}$ Teflon and silicone have both been previously used as permanent VF augmentation materials; they were discontinued owing to the significant immunologic/granulomatous responses seen in numerous patients. ${ }^{14,15}$ )

Some previous studies reported the efficacy of CaHA in Asian with short-term follow-up. Woo et al. reported that all of the voice outcomes, including videostroboscopic findings and acoustic, perceptual, and subjective evaluations were improved with vocal fold augmentation using CaHA. ${ }^{16)}$ Although Kwon et al. reported that CaHA can been found in computed tomography scan as long as 18 months after injection laryngoplasty, functional assessment was not performed and the subjective and objective analysis of voice cannot be evaluated. ${ }^{17)}$ Till recently, there has been no long-term studies evaluating the long-term efficacy of CaHA in injection laryngoplasty of Asian patients.

Foreign body reaction and biodegradation is key element of deciding the duration of the efficacy of injectable. Chetri et al. reported the development of localized foreign body giant cell reactions around CaHA particles, but no foreign body granuloma, in a canine model at 12 months of follow-up. ${ }^{18)}$ Because this was a limited foreign body reaction around injection material without granuloma formation, it could be considered to be a natural reaction to any synthetic material. Compensation of intrinsic and extrinsic muscles and adaptation of new environment after injection laryngoplasty may also affect the subjective and objective voice outcomes. Among the objective parameters in our study, closed quotient and mean flow rate was maximal at postoperative 3 months. This means that structural efficacy and biodegradation may be similar, while compensation can affect the continuous improvement of subjective and objective outcomes until postoperative 6 months.

Our study has limitation that the number of included patients was too small. However, CaHA was mostly beneficial and subjective and objective voice outcomes continuously improved until 6 months. We think that, for uncertain reason, Asian patients with unilateral vocal fold paralysis showed relatively slow improvement of subjective and objective parameters. CaHA can be safely used in Asian patients and the onset of maximal efficacy seems to be slow than other studies with Caucasian patients. For the better clarification, well designed study with more long-term follow and patients is needed.

\section{Conclusion}

Injection laryngoplasty using $\mathrm{CaHA}$ for unilateral vocal cord palsy can be a safe and effective method in Asian patients. However, for uncertain reason, the time for the maximal efficacy seems to be longer than other studies with Caucasian patients.

\section{REFERENCES}

1) Rosen CA, Thekdi AA. Vocal fold augmentation with injectable calcium hydroxylapatite: short-term results. $J$ Voice 2004;18:387-91.

2) Belafsky PC, Postma GN. Vocal fold augmentation with calcium hydroxylapatite. Otolaryngol Head Neck Surg 2004;131:351-4.

3) Chhetri DK, Jahan-Parwar B, Hart SD, Bhuta SM, Berke GS. Injection laryngoplasty with calcium hydroxylapatite gel implant in an in vivo canine model. Ann Otol Rhinol Laryngol 2004;113:259-64.

4) Caton T, Thibeault SL, Klemuk S, Smith ME. Viscoelasticity of hyaluronan and nonhyaluronan based vocal fold injectables: implications for mucosal versus muscle use. Laryngoscope 2007;117:51621.

5) DeFatta RA, Chowdhury FR, Sataloff RT. Complications of injection laryngoplasty using calcium hydroxylapatite. J Voice 2012;26: 614-8.

6) Won SJ, Woo SH. Calcium Hydroxylapatite Pulmonary Embolism after Percutaneous Injection Laryngoplasty. Yonsei Med J 2017;58: $1245-8$.

7) Rosen CA, Lee AS, Osborne J, Zullo T, Murry T. Development and validation of the voice handicap index-10. Laryngoscope 2004;114: 1549-56.

8) Kwon CH, Kim YH, Park JH, Oh BM, Han TR. Validity and reliability of the korean version of the MD anderson Dysphagia inventory for head and neck cancer patients. Ann Rehabil Med 2013;37: 479-87.

9) Rosen CA, Gartner-Schmidt J, Casiano R, Anderson TD, Johnson $\mathrm{F}$, Remacle M, et al. Vocal fold augmentation with calcium hydroxylapatite: twelve-month report. Laryngoscope 2009;119:1033-41.

10) Carroll TL, Rosen CA. Long-term results of calcium hydroxylapatite for vocal fold augmentation. Laryngoscope 2011;121:313-9.

11) Ford CN, Bless DM, Loftus JM. Role of injectable collagen in the treatment of glottic insufficiency: a study of 119 patients. Ann Otol Rhinol Laryngol 1992;101:237-47.

12) Laccourreye O, Papon JF, Kania R, Crevier-Buchman L, Brasnu D, Hans S. Intracordal injection of autologous fat in patients with unilateral laryngeal nerve paralysis: long-term results from the patient's perspective. Laryngoscope 2003;113:541-5.

13) Karpenko AN, Dworkin JP, Meleca RJ, Stachler RJ. Cymetra injection for unilateral vocal fold paralysis. Ann Otol Rhinol Laryngol 2003;112:927-34.

14) Nakayama M, Ford CN, Bless DM. Teflon vocal fold augmentation: failures and management in 28 cases. Otolaryngol Head Neck Surg 1993;109:493-8.

15) Rubin HJ. Pitfalls in Treatment Od Dysphonias by Intracordal Injection of Synthetics. Laryngoscope 1965;75:1381-\&.

16) Woo SH, Son YI, Lee SH, Park JJ, Kim JP. Comparative Analysis on the Efficiency of the Injection Laryngoplasty Technique Using Calcium Hydroxyapatite (CaHA): The Thyrohyoid Approach Versus the Cricothyroid Approach. Journal of Voice 2013;27:236-41.

17) Kwon TK, Lee JE, Cha WJ, Song CM, Sung MW, Kim KH. Serial computed tomographic and positron emission tomographic properties of injection material used for vocal fold augmentation. Laryngoscope 2013;123(10):2491-6.

18) Chhetri DK, Jahan-Parwar B, Hart SD, Bhuta SM, Berke GS. Injection laryngoplasty with calcium hydroxylapatite gel implant in an in vivo canine model. Ann Oto Rhinol Laryn 2004;113:259-64. 